vol. $33-n^{\circ} 2$ et $3 \mid 2017$

Dire la violence des frontières. Mises en mots de la migration vers l'Europe

\title{
Dire la violence des frontières dans le rapport de force que constitue la procédure d'asile. Le cas d'Ali, de l'Afghanistan en Belgique
}

Telling the Violence of Borders in the Power Relations that Constitutes the Asylum Process. The Case of Ali, from Afghanistan to Belgium

Contar la violencia de las fronteras en el equilibrio de poder representado por el procedimiento de asilo. El caso de Ali, de Afganistán a Bélgica

Jacinthe Mazzocchetti

\section{(2) OpenEdition}

Journals

Édition électronique

URL : https://journals.openedition.org/remi/8736

DOI : $10.4000 /$ remi.8736

ISSN : $1777-5418$

Éditeur

Université de Poitiers

Édition imprimée

Date de publication : 1 septembre 2017

Pagination : 91-114

ISBN : 979-10-90426-59-7

ISSN : 0765-0752

Référence électronique

Jacinthe Mazzocchetti, « Dire la violence des frontières dans le rapport de force que constitue la procédure d'asile. Le cas d'Ali, de l'Afghanistan en Belgique », Revue européenne des migrations internationales [En ligne], vol. 33 - n² et 3 | 2017, mis en ligne le 01 septembre 2019, consulté le 15 avril 2022. URL : http://journals.openedition.org/remi/8736 ; DOI : https://doi.org/10.4000/remi.8736 


\title{
Dire la violence des frontières dans le rapport de force que constitue la procédure d'asile. Le cas d'Ali, de l'Afghanistan en Belgique
}

\author{
Jacinthe Mazzocchetti ${ }^{1}$
}

\section{Introduction}

Dans un contexte de fermeture et de gestion sécuritaire des frontières, mais aussi de criminalisation des migrants, le registre de la suspicion occupe une place importante dans le regard porté sur les demandeurs d'asile, en quête de sécurité et de droits (Valluy, 2006). C'est dès lors dans un climat peu propice que ces derniers sont amenés à se raconter de façon recevable. La procédure d'asile repose principalement sur la narration d'une histoire consignée et analysée par les agents de l'État, en charge d'évaluer le degré de correspondance avec les critères de la convention de Genève. Cet examen sensé opérer une distinction entre les " vrais " et les "faux " réfugiés (Kobelinsky, 2012), repose sur une conception " archétypique rêvée " (Akoka, 2011) du réfugié. L'impasse est faite " sur la nature éminemment construite de la qualité de réfugié et éminemment politique de la convention " (Ibid. : 13), comme si les critères d'obtention n'étaient ni situés dans le temps, ni reliés aux contextes économiques, politiques et géostratégiques (Akoka et Spire, 2013).

À partir de I'histoire de vie d'Ali Y. ${ }^{2}$, demandeur d'asile afghan (entré mineur en Belgique en 2009, mais non reconnu comme tel), I'article entend souligner l'incidence, sur les parcours et les constructions de soi, des violences qui découlent des logiques sécuritaires de gestion des migrations. L'analyse portera plus particulièrement sur les procédures relatives aux demandes d'asile : demandes initiales, recours, refus, etc. Si un seul récit est ici mobilisé, les questionnements soulevés quant à l'usage du récit biographique dans l'examen des demandes d'asile sont le fruit d'un travail de terrain de plusieurs années auprès

1 Professeur d'anthropologie, Université catholique de Louvain, Membre du Laboratoire d'Anthropologie Prospective, Place Montesquieu 1, 1348 Louvain-La-Neuve, Belgique ; jacinthe.mazzocchetti@uclouvain.be

2 J'ai rencontré Ali par l'intermédiaire de son réseau de soutien en Belgique en la personne d'A.G. qui se reconnaitra et que je tiens à remercier pour la confiance qu'elle $\mathrm{m}^{\prime} \mathrm{a}$ accordée. Je remercie également les coordinatrices du numéro ainsi que les lecteurs anonymes de la revue pour leurs commentaires judicieux. 
de demandeurs d'asile, de réfugiés et de déboutés du droit d'asile en Belgique et à Malte. Les matériaux ethnographiques collectés résultent principalement d'observations participantes, de recueil de récits de vie et d'analyses de dossiers (Mazzocchetti, 2008, 2014 et 2017).

Après avoir expliqué succinctement le déroulé de la procédure d'asile en Belgique et débattu des limites qui découle de l'approche positiviste actuellement à l'œuvre dans l'examen des récits, j'exposerai l'histoire d'Ali telle qu'il me la racontée en 2016, avec les points d'insistance qui lui sont propres. Ayant eu accès à de multiples sources relatives à son histoire (les comptes rendus d'auditions, les dossiers établis par son avocat, les récits de personnes de son entourage, etc.), il me semble d'autant plus important de donner une place de choix aux mots qu'il a partagé avec la chercheure que je suis. Ce récit a été recueilli en français, langue qu'il maîtrise aujourd'hui, arrivé pourtant en Belgique seul et illettré. Dans un troisième temps, je discuterai de deux des éléments de contentieux relatifs à son histoire non plus partagée, mais déposée et disséquée dans le cadre de la procédure d'asile : la minorité d'âge et la détermination des origines. Enfin, la dernière partie sera consacrée à une réflexion sur la violence et "l'impérialisme des frontières " (Walia, 2015), qu'elles soient géopolitiques, ethno-raciales ou juridiques.

\section{Narration et procédure d'asile}

En Belgique, la procédure d'asile comprend deux étapes principales. La première consiste en l'enregistrement de la demande auprès de l'Office des étrangers $(\mathrm{OE})$, qui après examen, juge de sa recevabilité. Si tel est le cas, le dossier est transmis au service " asile " de l'Office pour ensuite être traité par le Commissariat général aux réfugiés et aux apatrides (CGRA) tandis que le demandeur a la possibilité d'être hébergé et nourri en centres d'accueil ouverts en attendant l'issue de sa requête. La deuxième étape se déroule donc au CGRA dont les agents sont habilités à examiner la demande et à accorder ou non un statut. La procédure consiste en une interview au cours de laquelle le demandeur expose son histoire, fait valoir son besoin de protection, et apporte les éventuelles preuves matérielles susceptibles d'appuyer sa démarche (papiers d'identification, coupures de presse, certificats médicaux, etc.). Cette interview peut être préparée avec un avocat qui est, en principe, présent au moment de I'audition. En cas de décision négative, un recours peut être introduit devant le Conseil du contentieux des étrangers. Ce juge administratif ne possède aucun pouvoir d'instruction de sorte que le recueil du récit est principalement effectué par le CGRA.

Selon les recommandations du Haut-Commissariat des Nations unies pour les réfugiés (HCR), les agents du CGRA évaluent « les déclarations et le besoin de protection du demandeur d'asile à la lumière de la situation objective dans le pays d'origine ${ }^{3}$ ". Ainsi, " les déclarations doivent être cohérentes et plausibles et ne pas être contredites par les informations générales et particulières connues

3 Cf. http://www.unhcr.org/fr/publications/legal/4ad2f7fa383/guide-procedures-criteresappliquer-determiner-statut-refugie-regard-convention.html (consultée le 13/10/2016). 
et pertinentes pour la demande ${ }^{4}$ ". Pour évaluer la crédibilité du demandeur $d^{\prime}$ asile et son besoin de protection, les agents s'appuient sur les informations à disposition concernant le pays d'origine, telles que les rapports des organes des Nations unies, des institutions du Conseil de I'Europe, d'ONG, etc. Le CGRA a également son propre centre de documentation et de recherches. Les rapports reposent rarement sur des informations de première main et sont souvent politiquement orientés. Les agents disposent en outre de peu de temps pour effectuer des analyses triangulaires et croisées. Ils interprètent dès lors de façon souvent intuitive la source qui leur parait la plus sûre (Gibb et Good, 2013).

Parmi les éléments qui influencent la prise de décisions des agents, il est probable que les discours et les orientations en termes de gestion des matières relatives à I'asile des responsables politiques dont dépend le CGRA doivent avoir une incidence. Si cet aspect mériterait une enquête en soi, à noter, pour le cas qui nous occupe, la lettre adressée parThéo Francken, le secrétaire d'État à I'Asile et à la Migration, en novembre 2015, aux Afghans ayant l'intention de faire une demande d'asile en Belgique, qui indiquait notamment :

"The Belgian government decided to stop taking asylum decisions for Afghans claiming to be non-accompanied minors, unless certain conditions are fulfilled. [...] The Belgian asylum authorities decided that, based on the actual situation in Afghanistan, it is not necessary to give a protection status to all Afghan citizens. Every asylum application will be investigated on its own merits. Some get a protection status, but others are refused. A rejection of your asylum claim will result in an order to leave the country within 30 days. If you don't leave voluntarily, then Belgium will return you by force to Kabul. This is a genuine message of the Belgian government ${ }^{5}$."

Cette lettre, a entre autres, été dénoncée par la Ligue des droits de l'homme comme étant la manifestation d'une dérive autoritaire et discriminatoire ${ }^{6}$. Elle est cependant révélatrice du contexte contemporain dans lequel les requérants sont amenés à plaider leur cause, et les agents à tenter d'évaluer leur " bonne foi " ainsi que les dangers réels qu'ils encourent à retourner dans leur pays. En juin 2016, une nouvelle lettre est envoyée aux demandeurs d'asile afghans résidant dans les centres d'accueil par le secrétaire d'État présentant notamment I'Afghanistan comme un pays sûr et encourageant le rapatriement volontaire ${ }^{7}$ :

\footnotetext{
4 Idem.

5 Cf. http://www.lesoir.be/1053655/article/actualite/belgique/2015-11-25/theo-francken-auxafghans-ne-venez-pas-en-belgique (consulté le 13/10/2016).

6 Cf. http://www.liguedh.be/2015/2455-lettre-de-theo-francken-aux-demandeurs-dasileafghans-une-derive-autoritaire-et-discriminatoire- (page consultée le 13/10/2016).

7 Cf. http://ptb.be/articles/francken-etend-sa-campagne-de-dissuasion-aux-refugiesafghans (page consultée le 13/10/2016).
} 
Figure 1 : Lettre envoyée aux demandeurs d'asile afghans résidant dans les centres d'accueil par le secrétaire d'État (juin 2016)

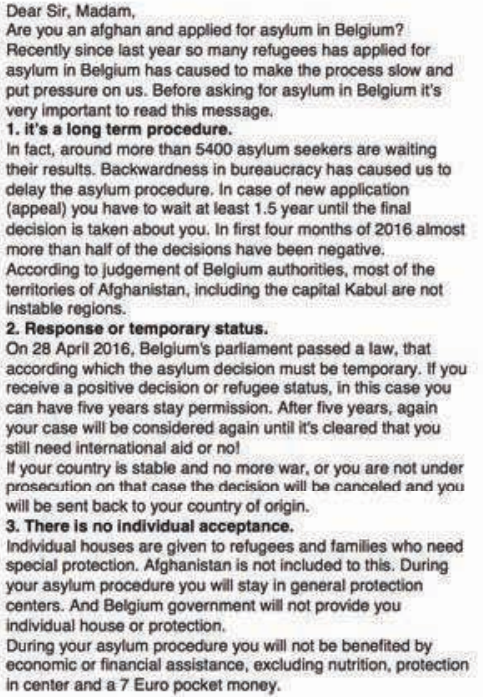

4. A long term procedure for family reunion. You can apply for a family reunion only when you have final positive decision. In this case, if you ask for family reunion, about another 9 months are needed for your case to be considered. In this period the documents of all members of your family will be considered very seriously. Any kind of take document or identity or wrong age will cause the refusal of your family reunion and the positive decision which has already given to you will be taken back. If you have got a final negative decision, in this case you are you have got a ina negativo decision, in this case you a obligated to ietur back to your country of orgin. If you (y) ou. If you deny the voluntary metum, on that case the government will force you to return back and in Schengen zone three years you wil nol be allowed to retum. Ladies and Gentlemen, frealize and understand that the given information to is not pleasant. I think it's very important for you to know, if you want to ask asylum in Belgium or whatever you expect, you must have exact and enough information about. you really dan't want to continue your asylum procedure in Belgium you can ask help from the government of Belgium in order to deport you back to Atghanistan for free. Also, free flight and tacilities until your return and other facilties during the flight and an amount of 500 Euro for each person will be paid. If you are interested you can contact your social assistant. If you want to start your personal work or business in Herat, Kabul and Jalalabad, the Belgium governments will financially help and advice you.

These facilitias are limited to only those Afghans who have approached jefore 01 June 2016 and are valid from 15 June 2016 till 15 July 2016.

Theo Francken,
Secretary of state on issues of immigrants and asylum seekers

Source : http://ptb.be/articles/francken-etend-sa-campagne-de-dissuasion -aux-refugies-afghans (consulté le 13/10/2016).

Ainsi, sur la base des informations disponibles, il s'agit, pour les agents, de vérifier l'adéquation entre les connaissances à leur disposition et le récit déposé. La crédibilité de la demande d'asile repose en grande partie sur l'analyse des récits à travers le prisme de critères de "plausibilité " et de "cohérence ". Faute de preuves matérielles, ou pour le moins de preuves considérées comme authentiques, l'enjeu de la narration est primordial. En effet, le contexte contemporain de suspicion influence également l'examen des éventuelles preuves matérielles " qui échappent rarement à une disqualification " (Belorgey, 2004 : 60 ) et sont souvent considérées comme étant des faux. Dès lors, pour obtenir un statut de protection, le requérant doit être en mesure de "travailler sa voix " (D'Halluin, 2006), c'est-à-dire de faire entrer son histoire dans les cadres de la recevabilité. La procédure d'asile est " une mise à l'épreuve " (Vrancken, 2011) de cet " autre " qui doit prouver qui il est, ainsi que le caractère légitime de sa demande. Pour le demandeur, il y a dès lors nécessité " de se dire ", mais plus encore de "se dire bien " : en usant du vocabulaire adéquat et en mobilisant le système de pensée qui l'accompagne ; de façon chronologique, précise et logique. Il importe de se mettre à nu en décrivant les violences subies et les craintes à venir. Les mots contraints le sont sous une forme très précise qui ne prend guère en compte la complexité des processus narratifs biographiques, mais aussi le fait que les référents culturels et les apprentissages scolaires ont une influence sur les capacités d'énonciation. 
Plusieurs recherches présentées ci-après, dans le domaine de la psychologie et de l'anthropologie notamment, soulèvent des questions clefs quant à la validité et au sérieux d'un usage juridique de la narration de soi dans le cadre de la procédure d'asile. Ces études mettent à jour différents éléments qui participent souvent de façon croisée au discrédit des récits examinés. Notons tout d'abord l'incidence sur le récit déposé du cadre institutionnel de l'asile, et les rapports de force propres à cette interaction singulière que constitue le moment de l'audition. Dans leur étude réalisée auprès de demandeurs d'asile et de réfugiés au Royaume-Uni, Bögner et al. (2010) analysent les facteurs qui influencent le dévoilement d'informations sensibles au moment des auditions. Ils décrivent notamment l'incidence des attitudes des agents du ministère de I'Intérieur en charge des interviews sur les paroles déposées, en particulier lorsqu'il s'agit d'événements traumatiques ou honteux. Ce qui est souvent qualifié de "mensonge par omission " est ici envisagé de manière plus complexe. Les demandeurs d'asile qu'ils ont rencontrés expriment de grandes difficultés à raconter les détails des expériences vécues, en particulier face à des agents aux attitudes sévères ou inquisitrices, peu propices à la confidence. Ces agents, en outre, leur rappellent bien souvent la police ou les milices des pays qu'ils ont fuis. Ils ne peuvent donc pas aisément leur accorder leur confiance.

Outre le paradoxe enjoignant aux demandeurs de raconter les détails de leurs souffrances et persécutions dans un cadre institutionnel peu propice, les entraves psychiques et culturelles relatives aux traumas ont une incidence forte sur les capacités à dire. L'étude de Vanoeteren et Gehrels (2009 : 504) est très explicite à ce propos : "Les mécanismes d'évitement, de refoulement et de confusion quant aux contenus les plus pénibles de l'expérience vécue font partie du tableau clinique régulièrement rencontré chez des personnes traumatisées ". Certaines atteintes sont parfois impossibles à exprimer, en tout cas dans le cadre imposé, notamment pour des raisons de honte ainsi que de crainte quant à l'usage potentiel de ces confidences en dehors du cadre de la procédure. Des vécus traumatiques sont également parfois inaudibles, car leur énonciation ne peut-être que fracturée, fissurée tout comme la personne qui tente de se raconter, qualifiée dès lors d'incohérente (Jacques et al., 2016).

Dans leur article intitulé " JustTell Us What Happened toYou: Autobiographical Memory and Seeking Asylum ", Herlihy et al. (2012) s'interrogent sur le fonctionnement largement aléatoire et subjectif de la mémoire. Ils mettent, d'une part, en évidence cette idée somme toute banale, mais évincée du fantasme d'objectivation qu'est la procédure : les souvenirs ont un caractère dynamique et évolutif. Ils ne sont que de pales reflets, toujours situés, des situations vécues. D'autre part, ils déconstruisent l'évidence de la mémorisation des noms, des dates et des lieux qui, en réalité, diffère fortement d'une personne à l'autre. L'énonciation détaillée et chronologiquement précise d'une expérience demande à être préparée. En outre, les repères marquants pour les uns, notamment sur la scène géopolitique, ne le sont pas forcément pour les autres. Les auteurs exposent également comment, contrairement aux idées reçues, avoir réellement vécu un évènement traumatique ne suppose pas de se souvenir des détails afférents. $\mathrm{Au}$ contraire, de nombreuses études, précisent les auteurs, mettent en évidence les effets de trouble et les incidences sur les capacités à se remémorer provoqués par les traumas. Les atteintes violentes ne sont pas forcément celles dont la mémoire garde une trace précise. 
S'ajoutent à ces différents éléments, l'enjeu des référents culturels. En prenant garde de ne pas tomber dans le piège culturaliste qui consisterait à essentialiser les différences et à expliquer les comportements par la culture en omettant l'incidence des contextes sociaux et interactionnels, il convient de noter que les implicites culturels des agents et des demandeurs jouent un rôle non négligeable dans l'ensemble du processus. Si les capacités à dire sont culturellement situées, les capacités à entendre des agents, par ailleurs en situation d'autorité, le sont également. Par quel biais, les mots déposés sont-ils reçus ? Faute de preuves considérées comme authentiques, faute de témoins, le jugement de crédibilité porté par les agents du CGRA sur le récit est principalement de l'ordre de la perception de véracité : le requérant connait-il les informations relatives à son pays à disposition des agents ? Le requérant parvient-il à énoncer clairement, sans contradiction, de façon répétée et détaillée les événements qu'il dit avoir subis ? Les réactions et les choix qu'il prétend avoir posés sont-ils cohérents?

Pourtant, les récits, à chaque fois singuliers, des demandeurs d'asile sont souvent impossibles à vérifier. Dans leur étude, Herlihy et al. (2010) s'intéressent dès lors aux critères mobilisés par les juges de l'immigration au Royaume-Uni pour évaluer le degré d'honnêteté des requérants. D'une part, ils mettent en évidence combien les connaissances sur lesquelles s'appuient les juges afin de repérer des indices révélateurs des comportements malhonnêtes sont culturocentrées. Ces derniers tiennent par ailleurs très peu compte des effets induits par les traumas. À titre d'illustrations, regarder ou pas dans les yeux peut avoir selon les cas des significations tout à fait opposées (honneur, hiérarchie, respect, malaise, etc.). D'autre part, les auteurs relèvent que, dans de nombreux cas, les juges se font en réalité une opinion en référence à leur propre perception du comportement adéquat et plausible relatif aux situations énoncées : qu'auraientils fait dans tel ou tel cas, et ce en lien avec leurs propres perceptions de l'attitude adéquate à adopter? La notion de " common sense " (Herlihy et al., 2010) - leur perception du "bon sens ", du " sens commun "- joue un rôle appréciable dans l'évaluation des dossiers supposément évalués selon une prétendue universalité et donc objectivité. Pourtant, comme le précise Good (2011: 81) dans une étude sur le rôle des narrations dans la procédure d'asile en Grande-Bretagne :

"For many asylum narratives, however, the cultural differences between teller and listener are such that common understandings cannot necessarily be assumed.

Constructing witness statements is crucial, therefore, because this process allows legal representatives to structure their clients' accounts according to the common sense expectations of western legal cultures. "

Ainsi, bien que la cohérence et la plausibilité des récits sont supposées être mesurées à l'aune des informations disponibles, les décisions prises par les agents reposent également sur le principe d' "intime conviction ". Notion qui, pour Greslier (2007), suite à ses enquêtes menées auprès de l'Office français de protection des réfugiés et apatrides (OFPRA) et de la Commission des recours des réfugiés (CRR), témoigne " de la parodie de justice dans laquelle la Commission des Recours des Réfugiés semble être prise ". Son étude l'amène en effet à mettre en évidence " la part d'arbitraire de l'État sur le traitement des demandes d'asile " et une orientation de plus en plus restrictive (Greslier, 2007 : 89). A contrario, dans sa recherche portant sur l'instruction de la demande d'asile 
en France et en Allemagne, Probst (2011: 69) décrit comment les agents rencontrés disent chercher " à éviter des appréciations démesurément subjectives par intime conviction ". "S'en tenir aux faits ", c'est également ce que m'expliquait un ancien agent du CGRA. Au final, comme le note Greslier, que le principe d'intime conviction soit assumé, ou, au contraire, qu'il demeure de l'ordre de l'impensé, comme dans le cas étudié par Probst : ce principe joue un rôle clef dans le tournant positif ou négatif que peut prendre la procédure. Que l'agent se replie derrière la notion ou qu'il croit pouvoir " s'en tenir au fait ", la prétention de la possibilité d'objectiver les situations est la même. D'un côté, il semble possible de s'appuyer sur des intuitions imaginées justes, car de "bon sens" et nourries d'informations brutes. De l'autre, les faits sont envisagés comme neutres, bruts, ayant une existence en propre en dehors des situations, des interactions et des sujets, etc. Cette approche positiviste du récit agrémentée des structures ethno-centrées et hégémoniques de pensée est au cœur de l'étude de cas proposée dans la suite cet article.

\section{Récit d'Ali : « La vie des Afghans, ce n'est pas une vie humaine "}

Dans un tel contexte de doute et de discrédit quant à la parole du demandeur d'asile, la valeur méthodologique des récits recueillis par les chercheurs se pose (Mekdjian, 2016). II s'agit de se démarquer d'une approche positiviste et de toute prétention d'exhaustivité. Le récit est ici appréhendé comme une narration située. II est ce qui vient faire rencontre entre le chercheur et la personne en demande d'asile, qui, en dépit du contexte, offre quelque chose de son histoire en partage, dépose des "paroles précieuses " (Métraux, 2007), sans maîtriser tout à fait ce qui en sera fait. Le récit est aussi un espace de reconstruction et sa visibilité peut faire office de contre-discours face aux discours politiques et médiatiques dominants (Eastmond, 2007). Si du côté des avocats et des personnes de confiance qui ont suivi le dossier d'Ali, il était nécessaire pour le suivi juridique de son dossier de se procurer des documents corroborant son récit, qui $\mathrm{m}^{\prime}$ ont par ailleurs été transmis ${ }^{8}$, la démarche ethnographique est autre. Elle n'a pas pour objet de dire le "vrai ", mais bien de mettre en tension des paroles, des cadres et leurs incidences en termes de violences. Le mensonge tel qu'abordé ici est entendu comme la conséquence d'un récit perçu comme non crédible et de ses effets. Le rapport de force observé s'inscrit dans des enjeux de pouvoir qui, à la fois, l'instituent, le dépassent et l'englobent.

\section{Un départ, des départs?}

Hazara, groupe minoritaire historiquement discriminé et cible des talibans, Ali est né dans un petit village des montagnes afghanes. Il était berger :

" J'avais onze ans quand j'ai quitté mon village vers I'Iran. Je suis parti avec mon cousin, du côté de ma mère. J'ai resté quelques années en Iran. Trois ou quatre fois, ils m'ont expulsé en Afghanistan. Je n'avais jamais pensé que j'allais vernir un jour en

Europe. C'était pour travailler, pour quitter mon village, pour ne pas rester toujours berger.

8 Les documents $\mathrm{m}^{\prime}$ ont été fournis par A.G., personne de confiance qui soutient Ali dans I'ensemble de ses démarches depuis plusieurs années, ainsi que par Ali. C'est également par l'intermédiaire d'A.G. que j'ai rencontré Ali. 
Et puis, j'avais des problèmes aussi avec les talibans. Quand j'étais petit, ils disaient tout le temps à mon père : "quand ton fils va grandir, on le prend avec nous". Souvent il y a des trucs comme ça."

La situation familiale dans laquelle il a grandi participe aussi de sa mise en route :

"Mon père a divorcé avec ma mère. J'étais trois ou quatre mois quand ma mère est partie. Je n'ai pas bu le lait de ma mère. La deuxième maman n'était pas très sympa, tout le temps ses enfants qui part à l'école. Et moi jamais. J'avais tout le temps des problèmes avec... "Va travailler, va faire ci, ça". Quand je dis : "je veux étudier", "non ne va pas étudier". Elle ne voulait pas que j'avance. Et puis mon père aussi, il s'est marié et puis il est parti loin, il n'est pas resté avec nous. Je ne pouvais pas aller chez ma mère et mon oncle, du côté de mon père, aussi tout le temps contre moi et qui me surveille. En fait, on

était contre la famille de ma mère. C'est pour ça, ma mère elle dit: "non, mon fils, il ne peut pas rester comme ça. Regarde comme il a grandi mon fils, regarde". C'est ça qu'elle a essayé de dire à son frère et, finalement, un jour, il m'a dit : "Ali il faut que je fasse quelque chose pour toi, mais ne le dis à personne. Un jour, je vais te ramener quelque part, tu pars d'ici". Il a dit: "je te ramène loin. Je ne sais pas où". Et puis petit à petit quand je suis parti, il a dit : "tu vas aller avec mon fils en Iran". "

\section{De I'Iran à la Grèce, en passant par la Turquie}

"C'est comme ça que je suis parti en Iran. J'ai trouvé un travail là-bas, j'ai travaillé dans le bâtiment. J'ai plusieurs fois été arrêté et expulsé en Afghanistan. C'est pour ça je ne suis pas resté en Iran aussi. Chaque fois que je gagne de l'argent, je perds la moitié, même plus pour les passeurs. Et puis mon oncle aussi m'a donné des forces. II m'a dit: "si vraiment tu veux aller quelque part, il faut que tu travailles, mais je vais t'aider". J'ai économisé l'argent petit à petit. Mon oncle, il a dit: "je fais confiance à Ali.

Tu vas en Turquie et puis en Grèce et puis en Italie".

Le passage de l'Iran à la Turquie, c'est pas facile. Il y a des montages que tu dois passer. II y a des mines. II faut faire attention de ne pas mettre tes pieds sur les mines. J'ai vu des gens qui a marché et qui a disparu, qui a explosé. J'ai resté aussi un mois, un mois demi dans une maison des passeurs. II te faut cacher, pas bouger, sinon voilà...

J'ai discuté avec un passeur qui parle ma langue, un Afghan. II dit: "oui, moi j'envoie les gens en Europe. Si tu veux, il y a plein de gens, mais je suis le meilleur". Tout le monde dit "je suis le meilleur". Finalement, il dit: "tu donnes 3000 euros et je te ramène jusqu'en Turquie. Et après, si tu veux décider d'aller encore plus loin, on est en contact, tout ça, je vais essayer de trouver. J'ai des personnes qui travaillent là-bas pour moi". Petit à petit, finalement, tu arrives jusque Turquie.

J'ai resté un mois en Turquie. II me donnait à manger, mais pas grand-chose. On était quinze. Je ne me souviens pas très bien, c'était il y a longtemps. Et puis on est venu jusqu'à Istanbul, il a fait un faux passeport. Parce qu'à Istanbul, il y a des contrôles tout ça et quand je montrais ça, il ne m'a rien dit. J'ai eu de la chance. Je suis passé. Et puis j'ai trouvé un passeur qui m'a ramené jusqu'en Grèce. "

\section{De la Grèce à I'Italie}

" Je suis passé par la mer. J'ai resté quinze minutes, vingt minutes maximum sur le bateau. J'ai arrivé dans une île, mais j'ai oublié le nom. Le matin, la police est venue avec un grand bateau. Certains qui savaient nager étaient déjà passer la nuit parce que vraiment ce n'était pas trop loin en fait. Une heure ou un peu plus, mais on dirait que l'eau 
est un peu calme. J'ai regardé, mais je ne sais pas nager et j'ai peur de l'eau. Certains sont passés pour ne pas prendre les empreintes. Moi je me dis, on n'a pas le choix et puis...

On m'a emmené dans une ville en Grèce et là, on est resté dans le commissariat de police en fait deux, trois jours. De là, chacun paye trente euros pour le ticket jusqu'à Athènes.

À Athènes, je suis resté six mois là-bas parce que je n'avais pas assez d'argent pour passer. J'ai demandé l'aide de mon oncle. Et il m'a envoyé un peu de l'argent. Quelques jours après, j'ai entendu des gens qui part à Patras. Patras, c'est un peu vers... Tous les bateaux qui part vers l'Italie en fait. Je me suis dit pourquoi donner l'argent aux passeurs si les gens partent gratuit. Et puis je suis parti là-bas. Non, mais c'est pas ça en fait. Tu pars gratuit et il y a plein de polices qui te frappent. Tu es dans la merde, quoi. Quand tu

pars, le camion, il ne te le laisse pas rentrer. Il y aussi là-bas des passeurs. II dit: "ici, c'est ma place. Cet endroit, c'est mon quartier. II n'y a personne qui peut monter dans le camion de cet endroit". Certains disent: "si tu pars de cet endroit, il faut que tu me payes".

Quand le camion s'arrête, des gens ouvrent la porte et directement cinq, six qui montent. S'ils ne te voient pas, tu passes et sinon ils t'arrêtent. J'ai vu un qui est monté, il a ouvert

la porte et un autre camion qui l'a écrasé. Vraiment en deux, sa tête explosée. C'était ça, on prend le risque. Patras, c'était comme ça. En Grèce vraiment, j'ai eu beaucoup de problèmes. C'est là-bas que j'ai fort senti que vraiment la vie, ce n'est pas facile. La vie des Afghans, ce n'est pas une vie humaine. C'est une vie d'animaux. Là-bas, j'ai vu des gens en train de pleurer et personne qui l'aide. Je n'ai pas assez d'argent, mais qu'est-ce que je dois faire?

Moi, j'étais caché plusieurs fois dans les camions. Et puis il m'a arrêté, il m'a frappé, les matraques et tout ça. Chaque fois, je dis : "non je dois passer, je ne vais pas rester ici toute ma vie courir après les camions". Et puis une fois, j'ai réussi. J'étais trente-six

heures dans le camion, on était à quatre en dessous des pastèques et puis après, de l'autre côté, il a contrôlé et il m'a renvoyé en Grèce. J'ai resté un mois dans le commis-

sariat de police. C'était comme un centre fermé en fait. J'étais trop loin, près de la Turquie. Je pensais qu'ils allaient me renvoyer. J'ai resté un mois sans douche, sans rien. Vraiment, je pue, c'est pire qu'animaux. Comme dans une ferme, c'était pire que ça. Pire que ça. Je dis: "qu'est-ce que je dois faire". Je gratte chaque fois et il y a des trucs qui mangent le sang quoi. La nuit, je n'arrive pas à dormir. La journée, je n'arrive pas à dormir.

Je dis : "je vais me tuer quoi, je vais me tuer, ça, ce n'est pas la vie".

Après un mois, il m’a laissé sortir, mais il m'a dit: "si on te voit encore au Patras, cette

fois-ci on te renvoie directement en Afghanistan, c'est comme tu veux. II est marqué interdit au Patras, regarde c'est marqué". Finalement je suis resté un mois et demi, puis

je suis revenu à Patras. Une fois, c'était dans une pompe à essence, un camion s'est arrêté, je dis : "c'est celui-là, je m'en fou, mais je dois rentrer dans ce camion-là", mais le camion était presque vide, il y avait juste des bidons remplis de pétrole de dedans, je dis : "comment je vais cacher". Le passeur dit : "va dans celui-là, comme c'est vide, peut-être ils ne contrôlent pas. Tu as ta chance. Essaye".

Et puis, je suis venu en Italie. Une fois loin du port, on a frappé sur la porte du camion et puis le chauffeur, il a appelé la police. La police est venue nous chercher et nous a amenés au commissariat. II a dit: "vous venez de quel pays, quel âge et tout ça". J'ai dit:

"j'ai seize ans". II dit: "non, tu es moins âgé, tu as quatorze ans, mais pas plus". Comme on ne parle pas très bien anglais, c'est difficile communiquer tout ça. Il a mis quatorze ans et il a appelé une famille d'accueil. " 


\section{Au centre fermé de Vottem (Belgique)}

En Italie, Ali économise progressivement de l'argent pour prendre le train en direction de la Norvège avec un autre jeune hébergé qui dit avoir un frère là-bas en mesure de les accueillir. II parvient également à recevoir le peu d'argent qu'il lui reste (200 euros). Dans le train, à Aix-la-Chapelle, il se fait arrêter par la police :

"II m'a dit: "tu veux rester en Allemagne ou pas ?" J'ai dit: "non, moi je veux aller en Norvège". Finalement, on m'a envoyé en Belgique parce que j'ai passé par la Belgique. Parce que ça apparaissait sur le ticket. Il a demandé l'âge, moi j'ai dit: "j'ai seize ans". Et puis après, je suis resté quinze jours dans centre fermé en Allemagne. II m'a ramené là-bas pour savoir j'ai quel âge. Et puis il me dit: "monsieur, tu as dix-huit ans, tu n'as pas seize ans". II a dit que je suis majeur, je n'ai pas de preuves que je suis mineur, j'ai pas de carte afghan, il m'a envoyé en Belgique. J'ai été à Vottem. À Vottem, il m'a demandé : "tu as quel âge", j'ai dit: "j'ai seize ans", mais il n'a pas accepté. Après j'ai été deux mois et demi à Vottem. II m'a dit: "dans centre fermé, tu as deux choix : soit tu demandes asile en Belgique, soit je t'envoie en Afghanistan". J'ai dit: "moi je ne veux pas rester ici, je veux aller en Norvège". II a dit: "il n'y a pas Norvège, il y a deux choix". Et puis finalement, j'ai demandé asile et j'ai reçu négatif. "

\section{Un récit « hors cadre »}

Bien que la question des frontières et des violences traversent l'histoire de vie d'Ali, dans cette troisième partie, j'analyserai uniquement les enjeux relatifs au récit soumis, dans le cadre de la procédure d'asile, au crible de la crédibilité. Comme exposé dans la première partie, au vu de l'impossibilité de la preuve, il s'agit surtout pour les agents de déterminer s'il y a mensonge ou non. En principe, le HCR recommande aux agents de tenir compte des vulnérabilités des demandeurs, telles que l'âge ou l'état psychologique (stress post-traumatique). Cependant, comme nous le verrons avec I'histoire d'Ali, être reconnu "vulnérable " découle de l'appréciation des agents. Ayant eu accès aux comptes rendus d'audition (demandes successives et recours), même si la parole de l'agent est indirecte, ce qu'il a retransmis des interviews et les rapports qu'il a écrits donnent un accès partiel à ses mots et aux schèmes de pensée qui les accompagnent. II ne s'agit pas, ceci dit, d'analyser les attitudes de cet agent en particulier, mais de mettre en exergue ce que ce cas donne à penser de la culture d'institution et de l'incidence du contexte de fermeture et de gestion sécuritaire des frontières sur les procédures.

Les extraits de documents officiels dans la suite de cette partie proviennent de la motivation de refus de la cinquième demande d'asile d'Ali en janvier 2015, reçue en novembre 2015, ainsi que du compte-rendu de cette audition. Je discuterai plus spécifiquement deux des points de contentieux explicitement énoncés dans les documents qui, à des niveaux différents, ouvrent à des questionnements importants quant à la politique contemporaine des frontières et de l'asile : la détermination de l'âge et de l'origine. La motivation du refus face à la dernière demande introduite par Ali est par ailleurs introduite en ces termes : " D'abord il faut insister sur le fait que vos première et quatrième demandes d'asile se sont terminées par le refus du statut de réfugié et le refus de protection subsidiaire 
parce qu'aucune foi n'a été accordée à votre prétendue origine de Jaghuri. Le CGRA a remarqué dans sa décision de refus du 01/02/2009 qu'il n'y a aucune crédibilité à votre prétendue minorité et que vous ne savez presque rien de la région d'où vous prétendez être originaire ".

\section{La " prétendue minorité "}

À son arrivée, alors qu'Ali dit avoir seize ans, les tests biométriques auxquels il est soumis ne le confirment pas de manière formelle ${ }^{9}$. En effet, les enfants migrants dépendent à la fois des législations sur l'immigration et l'asile ainsi que des législations sur la protection de l'enfance. Les mineurs et les majeurs ne jouissent pas des mêmes droits sur les plans de l'accueil, de la santé et de l'éducation scolaire. En cas de doute sur l'âge du requérant, des tests biométriques, notamment des tests osseux, bien que peu fiables et contestés, sont utilisés afin de déterminer l'âge des demandeurs d'asile (Vallet, 2009 ; Réseau Éducation sans Frontières, 2015). La question de l'âge vient faire frontière. Elle n'est pas une fin de non-recevoir quant à l'asile, mais elle participe de la logique de catégorisation à l'œuvre, divisant de manière prétendument objective les vulnérables, les légitimes, les ayants droit, et les " autres ", probables abuseurs.

Pour Ali, l'appréciation qui est faite des résultats des tests établit qu'il est âgé de dix-huit ans. Je parle " d'appréciation " car, outre les études qui remettent en cause la fiabilité de ces tests en particulier dans le cadre de la demande d'asile, suite aux deux examens passés : la tranche d'âge proposée par les médecins ayant examiné Ali se situe entre seize et vingt-six ans ${ }^{10}$.

Figure 2 : Extrait du rapport médical ayant pour objet la détermination de l'âge d'Ali

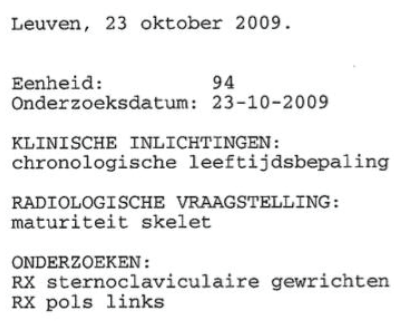

Source : Dossier d'Ali, communication personnelle.

La latitude d'interprétation est donc conséquente. Le déclarer majeur, contrairement à ce que l'usage de la science et de la biométrie pourrait laisser supposer, est le fruit d'une appréciation subjective. D'apparence très jeune, en Italie, c'est l'âge de quatorze ans qui avait été déterminé par les policiers qui I'ont intercepté quelques mois avant son arrestation en Allemagne.

9 La notion de minorité fait ici référence à l'âge du demandeur.

10 Le document présenté ci-après en reprend les conclusions. 
L'usage de la biométrie dans les procédures d'asile est loin d'être anodin. En effet, l'information biométrique est considérée comme " brute, objective, neutre, sans ambigüité "(Woodtli, $2008: 26)$ et donc non discutable. La prétendue objectivité de la science, la "vérité des corps " font ici office d'opérateurs de crédibilite : " the body is no longer the political locus in which power is manifested but the place in which individuals' truth about who they really are is experienced " (Fassin et D'Halluin, 2005 : 597). Ces pratiques mettent en lumière le cadre positiviste de la procédure ainsi que la dépolitisation, voire la déshumanisation, du requérant réduit à un corps qui, ici en l'occurrence, "parle mal ". Les contestations à l'égard de ces tests sont pourtant nombreuses, et ce, également, dans le chef des médecins :

"L'interprétation d'une radiographie n'est pas une méthode infaillible pour déterminer l'âge d'une personne. Cette interprétation requiert une expertise spécifique. [...] La technique de la détermination de l'âge osseux permet uniquement de déterminer l'âge du squelette ; la concordance avec l'âge civil du sujet est une appréciation diagnostique.

Différents facteurs (ethnique, génétique, endocrinien, socio-économique, nutritionnel, médical...) peuvent influencer la croissance d'un individu. [...] Les tables de maturation osseuse servant de références sont établies sur base d'une population déterminée, les plus utilisées reposent sur des populations blanches occidentales. Pour que la référence soit pertinente, le sujet auquel elles sont appliquées doit appartenir à la même population.

[...] L'estimation contient toujours un facteur d'imprécision, et ne peut dès lors aboutir qu'à fournir un intervalle de fiabilité. Le doute doit toujours profiter à la personne qui se déclare mineure. " "(Ordre des médecins, 2010)

Ces tests sont par ailleurs datés, mais surtout occidentalo-centrés, à la fois dans leur conception (c'est la science qui vient dire le vrai) et dans leurs données (c'est l'homme blanc occidental qui fait figure de référence dans les calculs effectués).

La détermination de l'âge a des implications très importantes sur la suite qui sera donnée à la demande d'asile d'Ali. Cette sentence des dix-huit ans le laisse seul face à la procédure dans un centre fermé pour adultes où il restera près de trois mois. II recevra comme seul appui une visite d'un avocat pro-deo ${ }^{12}$ qu'il $^{\prime}$ ne reverra pas par la suite. Sa première demande faite entre les murs du centre fermé de Vottem, sans aucune maîtrise des codes afférents, sans aucune préparation et sans soutien se soldera par un refus de droits qui vient d'être confirmé six ans plus tard :

"Le jour où le commissariat général est venu dans le centre fermé, il m'a posé plein de questions sur mon pays, c'était mon interview en fait, je n'avais pas d'avocat, rien. II y avait un traducteur, qui parle quatre langues différentes, pas le dari, mais une langue proche. " (Ali, 2016)

Dans les comptes rendus d'audition et la décision de refus, se donne à lire l'implicite dans le tri de l'essentiel d'une vie, comme si ce qu'il y avait à dire et

11 https://ordomedic.be/fr/avis/conseil/tests-de-determination-d-age-des-mineurs-etrangers-non-accompagnes (consulté le 13/10/2016).

12 Le système pro deo, appelé également Aide juridique, permet aux demandeurs d'asile de bénéficier de la gratuité totale ou partielle des services d'un avocat. 
à taire était de l'ordre de l'évidence :

"Durant l'audition préalable du 14/03/2014, vous rappelez que vous êtes originaire du quartier de Ghozba, village de Chebelpakht Pashi (CGRA 14/3/2014, p. 6). On vous a demandé pourquoi vous n'aviez pas donné ces renseignements plus tôt, vous avez déclaré de manière amusante qu'on ne vous l'avait pas demandé (CGRA 14/3/2014, p. §). Ce n'est pas sérieux."

Pourtant, comme I'expose Isnard (2011), dans un article réflexif sur sa pratique de juge :

"La parole exprimée devant les juges est presque toujours orientée. Peu importe que le réfugié soit effectivement prêt à "tout dire", ou même "tout montrer" : il ne le pourra pas. Le juge est le directeur actif du récit, même s'il se veut neutre et impartial dans le traitement de la demande. C'est lui qui interroge, ou laisse dire.

Rien n'est anodin. " (Isnard, 2011 : 111-112)

Dans le compte rendu de la première audition d'Ali qui n'a duré qu'une heure quarante-cinq, contrairement à la dernière audition de près de six heures, la plupart des réponses sont courtes. Elles se tiennent à ce qui a été demandé, sans explication ni contextualisation. En l'absence d'une préparation spécifique, puisque non reconnu mineur et donc non soutenu par un tuteur, de plus placé en centré fermé puisqu'arrêté en zone frontière ${ }^{13}$, il lui est de fait difficile d'imaginer ce qu'il y a lieu de dire. Cette attitude se voit dans l'argumentaire de la décision de refus qualifiée d' " amusante " et de " non sérieuse ", mettant en mots la violence du présupposé hégémonique d'un rapport identique aux connaissances, au récit et à la procédure qui est aussi violences des frontières, tant géopolitiques que culturo-livresques.

Pour en revenir à la question de l'âge, cette déclaration de majorité participe de l'arbitraire qui entoure la procédure d'asile et, de manière plus générale, les possibilités d'obtention de droits. Cette décision est aussi un point de basculement clef dans le parcours d'Ali, car la mise en cause de son âge, et son insistance à le clamer, le construisent non seulement " menteur ", mais aussi " de mauvaise foi ". Les documents qu'il pourra fournir par la suite, notamment sa tazkira ${ }^{14}$ reçue d'Afghanistan par son oncle, et qui lui donne raison sur son âge sont également non pris en compte, considérés comme faux. Et jusqu'au moment du dernier recours et du dernier refus, le critère d'âge est explicitement abordé :

"Vous maintenez en termes généraux que les talibans arrêtaient et tuaient les Hazaras (CGVS 14/3/2014, p. 3). Vous ne pouvez pas spécifier si votre départ pour l'Iran et donc ces combats avaient lieu pendant le régime des talibans (CGVS 14/3/2014, p. 3). Le fait de situer la raison d'un départ pour l'Iran pendant ou après la chute du régime des talibans constitue une différence importante qui n'aurait pas pu vous échapper si

13 Le rapport intitulé "Faire valoir ses droits en centré fermé " rédigé par un collectif $d^{\prime}$ associations (2008) est particulièrement explicite sur les difficultés que soulève la procédure d'asile depuis les Centres Fermés, notamment quant à l'absence de préparation et de soutien. Cf. http://www.aideauxpersonnesdeplacees.be/index_files/Publier/ aidejuridcentrefermintegr.pdf (consulté le 13/10/2016).

14 Pour rappel, il s'agit de sa carte d'identité afghane. 
vous quittiez effectivement l'Afghanistan à cause de l'intervention des talibans. L'excuse invoquée qu'à cette époque vous n'aviez que onze ou douze ans, doit en outre être sérieusement nuancée étant donné que le Service de Tutelle n'a pas accepté votre déclaration de minorité lors de votre arrivée en Belgique (CGVS 14/3/2014, p. 2). "

Cet extrait est intéressant à double titre, notamment concernant la question de l'âge. Les fragilités d'Ali, qui ont une incidence sur sa capacité à se remémorer, ne sont à aucun moment prises en compte. De même, la complexité de la situation qui prévaut en Afghanistan, la spécificité de sa trajectoire constituée de plusieurs allers-retours entre l'Afghanistan et I'Iran avant de rejoindre la Turquie et par la suite l'Europe, sont autant d'éléments ignorés par les agents. Pour l'examinateur, il n'était pas aussi jeune qu'il le prétend. Dès lors, l'âge ne peut pas être une excuse. Des dix-huit ans établis par des tests par ailleurs contestés, plutôt que des seize ans de sa carte d'identité afghane, il est considéré comme adulte et peu enclin à coopérer. Ses vulnérabilités potentielles sont évincées. Pourtant, même en accordant foi à l'appréciation subjective des tests, Ali aurait eu treize ans lors de son départ définitif, illettré, berger des montagnes, coupé des médias et de son père, parti depuis longtemps. D'autre part, la situation vécue par Ali met particulièrement bien en exergue le sentiment d'évidence de I'accès à l'information "cela n'aurait pas pu vous échapper " et l'imaginaire d'un départ du type action-réaction, ce qui, dans de nombreux cas, et ici précisément, est loin d'être effectif. C'est sur cet élément que je vais $m$ 'arrêter dans le point suivant.

\section{La " prétendue origine "}

L'histoire d'Ali est également considérée comme non crédible parce qu'il y a soupçon, je cite, de "fraude à l'origine ". Ce soupçon repose en grande partie sur la confrontation de deux rapports à l'espace distinct : d'un côté, l'espace vivant, et de l'autre, l'espace théorisé de la cartographie. La notion d' " espace vécu "(Frémont, 1974) n'est pas prise en considération, c'est un rapport à l'espace objectif et objectivable qui est attendu des agents, alors même qu'il ne correspond pas au rapport vivant que les hommes ont à leur environnement. Ce qui est connu d'Ali, c'est la vie à la montagne, le flanc sud et le flanc nord, les implications familiales des enjeux fonciers, plutôt que le nom des provinces et de leurs chefs-lieux. Au moment de la traversée des continents et des pays, celui qui sait la route, c'est le passeur et c'est d'ailleurs pour cela qu'on le paye relativement cher.

La vérification de l'appartenance et du chemin suivi se fait à partir d'un rapport livresque aux connaissances. Dans l'argumentaire du refus reçu par Ali en 2016, il est indiqué :

"Vous ne connaissez aucun autre district de la province de Ghazni, vous n'avez vraisemblablement jamais entendu parler du nom du district central de Jaghuri (Sangi

Masha), vous ne connaissez aucune des provinces frontalières de Ghazni et votre connaissance de la situation politique et sociale dans la région était quasi inconsistante. "

Le fait d'avoir face à soi un jeune homme, berger et analphabète à l'époque, ne semble aucunement recevable. Ali m'explique : 
"Je n'ai pas été école, je ne connais pas les chefs-lieux des provinces, en plus j'étais dans mon petit village et je ne suis pas sorti ailleurs. Quand j'ai sorti, c'est pour aller en Iran. J'ai dit tout le trajet, que j'ai passé tout ça, à quel endroit, à quel endroit. II a dit: "tu ne connais pas le chef-lieu de ton province, tu ne viens pas de là-bas". " (Ali, 2016)

En effet, les connaissances qu'Ali a de son milieu ne correspondent pas aux connaissances consignées. S'il mentionne bien être passé par Sangi Masha, il ne sait pas à l'époque qu'il s'agit d'un chef-lieu :

"Chaque fois, il me demande: "où est le chef-lieu ? Combien de provinces ?" Je ne sais pas. Moi je ne sais pas ce que c'est chef-lieu en fait. " (Ali, 2016)

Par ailleurs, Sangi Masha, qui est en réalité un village, était à plus de dix heures de marche du lieu de résidence d'Ali qui, avant d'aller en Iran, n'avait jamais quitté son village.

A contrario, le nom des nombreux petits villages qu'il cite n'apparaissent pas sur les cartes à disposition :

"S'il me dit: "donne-moi, tous les noms à côté de ton village", ça je connais, mais il ne le voit pas. C'est trop petit. Il regarde sur Internet et il n'indique pas. " (Ali, 2016)

Et en effet, sans vouloir à nouveau jouer ici le jeu du "vrai ", en préparation de sa nouvelle demande, la personne de confiance qui s'occupe de lui depuis maintenant plusieurs années a réussi à se procurer d'anciennes cartes soviétiques ${ }^{15}$ sur lesquelles apparaissent les villages nommés par Ali, ce qui démontre une connaissance très précise des lieux, mais une connaissance qui ne rentre pas dans les codes attendus:

"Les villages sont très petits, sans eau ni électricité. Personne qui n'a pas vécu là-bas ne pourrait citer ces villages. " (Ali, 2015)

Ces pièces ajoutées à son dossier, tout comme sa tazkira, ainsi que la carte d'identité et le carnet militaire de son père, ne permettront pas de réhabiliter son récit :

"Ainsi vous déposez un document du service militaire de votre père et deux pièces concernant l'engagement de votre père comme mudjadhin. Aucun des documents

apportés n'a de rapport avec votre situation personnelle et ne peuvent donc pas servir de preuve à vos déclarations concernant votre séjour en Afghanistan et en Iran. "

Ce qui pour Ali sera extrêmement déstabilisant car les démarches pour obtenir ses documents ont été longues et complexes et il espérait que le papier allait enfin faire preuve.

"La fraude à l'origine " et, dès lors l'absence de danger réel, sont également alimentées par l'impression de flou, du côté du CGRA, à propos des circonstances du départ d'Ali. Dans le rapport, on peut lire :

15 Cf. https://mapstor.com/map-sets/country-maps/afghanistan.html (consulté le 13/10/2016). 
"En même temps, dans la décision du CGRA dans le cadre de votre première demande d'asile, il a été établi que vous n'avez pas déposé de déclarations crédibles sur

le moment et les circonstances de votre départ pour l'Iran. "

Le départ est ici conçu comme un moment bien distinct, relatif à un événement précis, avec un avant et un après clairement identifiables. Cependant, la réalité est souvent plus complexe, à la fois plus diffuse et aléatoire :

" J'étais très jeune, je vivais en permanence seul dans les montagnes et j'étais illettré. Je savais qu'il y avait du danger, tout le monde avait peur, mais je ne connaissais rien des changements de régime. Tout ça me dépassait complètement. Comment aurais-je pu savoir quand les talibans sont arrivés au pouvoir et quand ils l'ont quitté ? Par ma belle-mère qui était tout aussi ignorante que moi ? Par la radio ou la télévision ? Nous n'avions pas l'électricité. [...] II n'y a pas eu de circonstances particulières à mon départ. Un jour mon oncle est venu me voir en disant qu'il allait faire quelque chose pour moi. Quelque temps après, il m'a dit que j'allais partir avec son fils. Je n'ai pas trop posé de questions et nous sommes donc partis ensemble, en cachette pour que mon père ne le sache pas. " (Ali, 2015)

De manière plus générale, malgré des périodes d'accalmies, la violence est quotidienne en Afghanistan depuis des décennies et la mise en route n'est pas forcément le résultat de circonstances précises (Javid, 2016). Monsutti (2009), dans son analyse des "réseaux migratoires afghans", met bien en exergue le non-sens d'une distinction radicale entre circulations volontaires ou forcées. Les mobilités et le climat d'insécurité sont à réinscrire dans une histoire longue qui rentre difficilement dans les cases de la procédure. Dans le cas d'Ali, comme dans de nombreux récits de demandeurs d'asile, il n'y a pas d'événements " mises en route ", mais un parcours de mouvements successifs en recherche de sécurité physique et économique. Le "moment et les circonstances " de son départ, mais aussi les craintes relatives à un retour aux pays sont multiples. Ali évoque des raisons d'ordre politique (les talibans, le danger diffus, les engagements politiques de son père, le fait d'être Hazara, etc.), des raisons d'ordre familial (les tensions entre la famille de son père et de sa mère, la peur ressentie face à son oncle paternel vraisemblablement violent, le décès de ses parents, etc.), mais aussi la désespérance d'une vie insécurisée et sans avenir, que ce soit en Afghanistan ou en Iran. Son départ est de plus jalonné d'étapes, ce qui en rend l'énonciation chronologique et événementielle pratiquement impossible. II a en effet fait plusieurs aller-retours entre l'Afghanistan et I'Iran avant, suite aux conditions de vie et aux violences subies en Iran (Monsutti, 2009), de se décider à partir vers l'Europe.

\section{Devenir un « sale menteur "}

Dans cette dernière partie, j'aborderai de manière plus spécifique la violence que constituent les rapports détaillés de refus qui viennent imprimer la privation de parole et le déni d'humanité. J'analyserai notamment les effets performatifs du discours de l'Office des étrangers "Vous mentez " qui finissent par instiller le doute dans le chef même du requérant, voire le sentiment progressif de ne plus exister. 
Si le fait d'être considéré comme non crédible, voire être accusé de mensonge, a des implications en termes d'accès aux droits, les incidences identitaires sont également importantes. Outre l'anxiété que provoque le moment d'incertitude qu'est la procédure d'asile (Vanoeteren et Gehrels, 2009) ; à la fois le déroulé de l'interview et la sentence du refus ont des conséquences psychiques et identitaires sur les requérants. Pour Ali, ce qu'il est, est nié, rejeté, et tous les documents qui appuient sa demande sont évincés :

" "Tu es menteur, tu es sale menteur", c'est ça qu'ils disent chaque fois. Une fois, il m'a demandé: "si tu nous dis la vérité, d'où tu viens vraiment, je te donne cinq ans de papiers tout de suite". Je ne savais pas ces gens-là, ils pensent comme ça. Si je savais, j'allais dire directement, mais je ne sais pas. Ils posent des questions, je donne des réponses. "Tu habites où ?" Ils ne disent rien, mais après à la fin, tu reçois négatif. Mais il faudrait vérifier sur Internet. Pour lui aussi, il a un doute, mais il faut demander. " (Ali, 2016)

Dans sa lettre de réclamation à destination du CGRA, la personne de confiance qui était présente à l'audition insiste sur cette réduction de l'autre, du demandeur à l'état de menteur. J'ai été "frappée, si pas choquée ", dit-elle, "par la tournure de l'interview ":

"L'agent a demandé à plusieurs reprises et avec beaucoup d'insistance à Mr. Y. de dire la vérité comme si ses réponses ne l'étaient pas... Après un quart d'heure environ, il s'est levé en demandant à Mr. Y. de réfléchir pendant dix minutes ; il s'est aussi adressé

à l'avocate afin qu'elle le raisonne et qu'il lui donne la "bonne" version des faits. II lui a conseillé à quatre reprises de dire clairement qu'il était Iranien ou Pakistanais et que, de cette manière, il recevrait le titre de réfugié. Enfin, Mr. Y., en répondant à une question, a fait référence à son âge mentionné sur sa tazkira et il s'est fait recadrer sévèrement en disant qu'il n'a pas cet âge. "

Bien entendu, il ne s'agit pas de se focaliser sur l'agent qui semble peu enclin à revenir sur sa première idée, sa probable "intime conviction ". II s'agit plutôt, au travers de ce cas, de réfléchir aux enjeux plus globaux de violences et de mise à mal du droit d'asile qui se donnent ici à voir. En effet, ce cas donne à penser le déroulé de la procédure et ses évidences : malgré des éléments potentiellement déroutants, rien de ce qui est amené au dossier ne vient ré-ouvrir un droit à l'écoute et, potentiellement, à la reconnaissance. Classé " menteur ", Ali est exclu d'emblée du droit, mais aussi, d'un rapport d'égalité. C'est donc bien de I'analyse d'un processus et de la violence de son ethnocentrisme qu'il s'agit :

"Comme vous n'avez pas raconté la vérité au sujet de vos véritables lieux de séjour pour votre départ vers la Belgique, le commissaire général s'est trouvé dans l'ignorance quant au pays vu en dernier où un besoin éventuel de protection devrait être requis, le lieu et les circonstances que vous avez effectivement vécus avant votre arrivée en

Belgique, de même que concernant les raisons effectives que vous avez présentées pour quitter ces lieux. Étant donné que vous avez délibérément caché la vérité (les vraies circonstances) sur ce point qui concerne le noyau de votre récit et de votre demande d'asile, vous avez, par conséquent et par votre propre fait, rendu impossible l'examen de l'existence d'une éventuelle crainte fondée, relative à des poursuites ou d'un réel risque de graves dommages. " (Rapport de refus, 2016) 
L'histoire déposée ne rentre pas dans les cadres ni dans les cartes. Les os radiographiés ne donnent pas les bonnes réponses et établissent le doute et l'exclusion. Davantage que le reflet des droits de l'homme, la procédure devient le reflet d'un contexte suspicieux et violent à l'égard des ressortissants étrangers des pays tiers et non détenteurs des codes de la " mondialité " (Ollivro, 2009). Le récit manqué car manquant devient la balise du reste du chemin à parcourir. Les imprécisions et les silences sont lus en termes d'omissions volontaires et de mensonges. Le contentieux à l'œuvre est pourtant de l'ordre du "double bind" (Bateson, 1995), car l'obligation de se " dire bien " est assortie de son impossibilité effective. La recherche de la "Vérité " dans sa conception ethno-centrée et positiviste, est une fabrique d'exclusion qui ignore tout des manquements à l'œuvre : ceux du requérant tout comme ceux des agents. En effet, de leur part, il ne peut être question que d'imaginaires de compétences face à la " pénurie permanente d'informations " (Probst, 2011 : 155) et à la complexité des enjeux narratifs et mémoriels. Le cadre tel qu'établi ne permet ni justesse, ni justice. D'autant que les éléments narratifs racontés le sont en réponse à des questions largement standardisées et orientées dans une perspective d'évaluation de la plausibilité du récit. Procédé en contraction avec un mode relationnel d'énonciation plus favorable à la remémoration et à la confidence (Good, 2011).

Une démarche qui reconnaitrait que les récits, tous comme les avis, ne sont que bricolages, permettrait peut-être une pensée véritablement critique de la procédure. Cette perspective supposerait une rupture épistémologique où les " faits" ne seraient plus considérés comme de la " matière brute ", ayant une existence en soi, mais comme le résultat d'une expérience incorporée et enculturée, et, dès lors toujours située. Comme l'énonce Isnard (2011), de sa place de juge :

" Simplement, puisqu'il est peut-être arrivé quelque chose, ce qui est dit ne doit pas être irréel. II est seulement nécessaire que le juge et le réfugié puissent adhérer, ne serait-ce qu'un instant, à ce qui est raconté par l'un, transcrit et interprété par l'autre. J'émets donc l'hypothèse que pour être en mesure de répondre à la souffrance de l'autre et de la reconnaître, pour permettre l'accès à la loi et son avènement, il faudrait d'abord suspendre ses préjugés, prendre la place de cet autre, devenir cet autre, puis reprendre sa place ; sortant de la souffrance comme d'une pièce, s'en distancier ; pouvoir penser un récit ou une identité en termes de fiction, sans que ce récit ou les protagonistes en cause s'en trouvent disqualifiés ipso facto. " (Isnard, 2011 : 113)

Une attitude davantage empathique et décentrée ne rendrait pas la procédure objective, mais permettrait peut-être, bien que ce ne soit pas l'objectif premier de l'institution, de réduire la violence de la rencontre en facilitant l'écoute, et, dès lors de reconnaitre quelque chose de I'humanité de l'autre. À l'encontre d'une approche positiviste, le regard porté du côté de la vérité du sujet en contexte donne accès aux blessures psychiques et morales vécues, tout autant que celles relatives à la procédure. Dire de l'autre qui joue sa vie qu'il est un menteur n'a rien d'anodin. Le discours ici posé est un discours à conséquence, où le poids des mots est effectif et tranchant, et dont les effets débordent de loin la sentence exprimée dans les rapports d'audition. II est parfois difficile d'y démêler l'illocutoire du perlocutoire, pour reprendre le débat auquel se livre Butler (2004), tant le " dire, c'est faire " (Austin, 1991) a des implications à la fois en termes de droits, et donc de sentences, et en termes identitaires : la fin de non-recevoir 
place le demandeur en dehors du droit, tandis que la qualification insistante de " menteur " a des effets de déstabilisation voire de destruction psychique. Le déni de nationalité et donc de crédibilité a une incidence sur le soi : "Tu n'es pas qui tu prétends être ", mais "Qui suis-je alors ?", se demande avec instance Ali :

"Ils disent que je ne suis pas Afghan. Je suis quoi alors ? Dis-moi ce que je suis alors! Ils disent que je suis né en 1991 et ma tazkira dit que je suis né en 1993, mais ils disent que ma tazkira est fausse. Alors je suis quoi moi ? Je suis pas Afghan, je suis pas né en 1993, alors je fais quoi ?" (Ali, 2013)

Ceci dit, les " capacités d'agir " (Butler, 2005) ne sont pas pour autant évincées. Le " pouvoir des mots " (Butler, 2004) est aussi du côté du demandeur qui dit sa vie pour tenter de la sauver, et qui peut également trouver à se reconstruire au travers d'autres scènes où déposer son histoire malgré les atteintes de la procédure. Ainsi, si la sentence juridique et le déni d'identité détruisent, les mots partagés ont aussi un effet potentiel de reconstruction psychique et de reconnaissance autre que juridique. Dans le cas d'Ali, son récit entendu et reconnu d'abord sur la scène intime de la famille qui l'a accueilli, ensuite dans des espaces plus ouverts, tels que des écoles, et enfin de façon publique via une vidéo réalisée par un comité de soutien médiatisant son histoire, postée sur Facebook et visionnée plus 20000 fois en quelques jours, participe d'une estime de soi retrouvée. Le récit a alors un caractère reconstructeur et permet, à certaines conditions, de retrouver du sens et de la confiance face à l'effraction du sujet et à son émiettement, de sortir dès lors de la non-existence, du "Qui suis-je alors ? ", "Que reste-t-il de moi ? ". Se joue quelque chose de l'interconnaissance, et dès lors de la reconnaissance (Ricœur, 2004). Ces prises de parole risquées, depuis sa (non-)place de "résident dit illégal ", sont aussi du côté de la " puissance d'agir " du langage (Canut, 2012). Elles viennent faire résistance face à un système de fabrique et de légitimation des exclusions.

\section{Conclusion}

Ali a expérimenté la violence des frontières sous de multiples formes toute sa vie. De la matérialité des frontières (la vie en Afghanistan, les conditions de séjour en Iran pour les Hazaras, les violences policières et l'enfermement ; les passeurs et la dangerosité des passages depuis I'Afghanistan vers I'Iran, la Turquie, la Grèce, I'Italie, l'Allemagne et la Belgique ; les morts laissés sur la route, etc.) à la procédure, se donnent à voir la production de catégories et leurs effets. D'un côté, les biens nés et les légitimes aux droits de l'homme et de l'autre, les menteurs, au mieux les victimes, les en deçà, les moins humains que les autres. Car en effet, la violence des frontières est aussi imprimée dans les représentations et dans les corps. Elle trouve également à s'exprimer au travers des évidences et des attitudes des agents, petites mains d'un système qu'ils reproduisent et qui trouve ainsi à se légitimer. La "Vérité " qu'ils traquent n'est que le reflet des "régimes de vérité " actuellement légitimés dans notre société, la "Vérité " qu'ils traquent n'est pas exempte de rapports de force et d'effets d'autorité (Foucault, 2011 ; Hardy, 2003).

Outre une attention aux récits singuliers des demandeurs, l'analyse anthropologique invite à décoder la " fiction discursive " (Hardy, 2003 : 482) à l'œuvre 
dans la procédure d'asile. Elle met en lumière l'épistémologie positiviste, ethnocentrée et impérialiste/paternaliste qui encadre la procédure. Au cours de ce processus, l'agent, détenteur de la norme, est habilité à dire le vrai du faux non seulement de la demande, mais également de celui qui la dépose, avec effet de négation du sujet. Si l'interaction n'est pas unidirectionnelle et que l'ethos de héros ordinaire et de résistant nait des violences des frontières successives, les rapports de force n'en restent pas moins tout à fait déséquilibrés. Le savoir juste, pour le moins considéré comme tel, est ici dans les mains de ceux qui jugent (sans par ailleurs être formés pour le faire), plutôt que dans celles de ceux qui ont expérimenté le monde.

Ultime atteinte, car elle imprime le déni des violences qui l'ont précédée, la procédure est dans de nombreux cas une scène de non-rencontre. L'impensé des rapports de force qui s'y déploient et des confrontations culturelles qui $s^{\prime} y$ jouent est le miroir de la violence du contexte contemporain pour les " vagabonds " comme les nomme Bauman (2010), les laissés-pour-compte du monde globalisé. La scène de l'asile contemporaine légitime et reproduit un système de catégories d'asymétries, à la fois géopolitiques, juridiques, culturocentrées. S'y donne à voir un positivisme impérialiste quant au bien-fondé du rapport sédentaire et nationaliste à la terre, avec un évincement du caractère international des conflits. Les impasses mises ici en lumière soulèvent des interrogations quant à la possibilité d'une " cosmopolitique " nourrie des expériences de ceux qui déjà la vivent (Agier, 2016) : comment transiter d'un monde de frontières, de murs, de barbelés à une "pensée du passant, du passeur, du passage, du passager " (Mbembe, $2016:$ 175-176), à une " pensée frontalière ", qui permettrait " une reprise/redéfinition des notions de citoyenneté, de démocratie, de droits de l'homme, d'économie, de politique, au-delà des définitions étroites imposées par la modernité euro-centrée (Grosfoguel, $2006: 65$ ) " et où serait " possible une réelle communication et un dialogue horizontal entre les peuples du monde " (Ibid. : 67) ? 


\section{Références bibliographiques}

Agier Michel (2016) Les migrants et nous. Comprendre Babel, Paris, CNRS éditions, $57 \mathrm{p}$.

Akoka Karen (2011) L'archétype rêvé du réfugié, Plein droit, 90 (3), pp. 13-16.

Akoka Karen et Spire Alexis (2013) Pour une histoire sociale de l'asile politique en France, Pouvoirs, 144 (1), pp. 67-77.

Austin John Langshaw (1991 [1962]) Quand dire c'est faire, Paris, Seuil, 202 p.

Bateson Gregory (1995 [1972]) Vers une écologie de l'esprit, Paris, Seuil, 299 p.

Bauman Zygmunt (2010 [1998]) Le coût humain de la mondialisation, Paris, Fayard/Pluriel, 208 p.

Belorgey Jean-Michel (2004) L'asile et l'intime conviction du juge, Plein droit, 1 (59-60), pp. 59-61.

Bögner Diana, Brewin Chris and Herlihy Jane (2010) Refugees' Experiences of Home Office Interviews: A Qualitative Study on the Disclosure of Sensitive Personal Information, Journal of Ethnic and Migration Studies, 36 (3), pp. 519-535.

Butler Judith (2005 [1990]) Trouble dans le genre. Le féminisme et la subversion de l'identité Paris, La Découverte, 284 p.

Butler Judith (2004 [1997]) Le pouvoir des mots. Politique du performatif, Paris, Éditions Amsterdam, $287 \mathrm{p}$.

Canut Cécile (2012) Transversalités langagières. Quelques notes pour une anthropologie des pratiques langagières, in Jean-Marie Prieur et Martine Dreyfus Éds., Hétérogénéité et variation Perspectives sociolinguistiques didactiques et anthropologiques, Paris, Michel Houdiard, pp. 87-95.

D'Halluin Estelle (2006) Travailler sa voix ou comment rendre sa demande d'asile audible, Le Croquant - Sciences humaines, art, littérature, 51-52, pp. 13-22.

Eastmond Marita (2007) Stories as Lived Experience: Narratives in Forced Migration Research, Journal of Refugee Studies, 20 (2), pp. 248-264.

Fassin Didier and D'Halluin Estelle (2005) The Truth from the Body: Medical Certificates as Ultimate Evidence for Asylum Seekers, American Anthropologist, 107 (4), pp. 597-608.

Foucault Michel (2011) 1970-1971 : Leçons sur la volonté de savoir, Paris, Gallimard, $318 \mathrm{p}$.

Frémont Armand (1974) Recherches sur l'espace vécu, Espace géographique, $3(3)$, pp. $231-238$.

Gibb Robert and Good Anthony (2013) Do the Facts Speak for Themselves? Country of Origin Information in French and British Refugee Status Determination Procedures, International Journal of Refugee Law, 25 (2), pp. 291-322.

Good Anthony (2011) Tales of suffering: Asylum narratives in the refugee status determination process, West coast line, 68, pp. 78-87.

Greslier Florence (2007) La Commission des Recours des Réfugiés ou "I'intime conviction " face au recul du droit d'asile en France, Revue Européenne des Migrations Internationales, 23 (2), pp. 107-133. 
Grosfoguel Ramón (2006) Les implications des altérités épistémiques dans la redéfinition du capitalisme global. Transmodernité, pensée frontalière et colonialité globale, Multitudes, 26 (3), pp. 51-74.

Hardy Cynthia (2003) Refugee Determination. Power and Resistance in Systems of Foucauldian Power, Administration \& Society, 35 (4), pp. 462-488.

Herlihy Jane, Gleeson Kate and Turner Stuart (2010) What assumptions about Human Behaviour Underlie Asylum Judgments?, International Journal of Refugee Law, 22 (3), pp. 351-366.

Herlihy Jane, Jobson Laura and Turner Stuart (2012) Just Tell Us What Happened to You: Autobiographical Memory and Seeking Asylum, Applied Cognitive Psychology, 26, pp. 661-676.

Isnard Hélène (2011) Le récit du réfugié est-il une fiction ?, Topique, 1 (114), pp. 107-114.

Jacques Paul, Khanian Nouné Kara et Xhema Elsa (2016) La guerre... des années après. Une pratique à plusieurs voix, in Luc Snoeck et Altay Manço Dirs., Soigner I'autre en contexte interculturel, Paris, L'Harmattan, pp. 155-164.

Javid Mohammad Ismail (2016) Le flux migratoire afghan. Une nation soumise au vagabondisme, Bruxelles, Siréas, 16 p., [en ligne]. URL : http://www.sireas.be/ publications/analyse2016/2016-01int.pdf

Kobelinsky Carolina (2012) Sont-ils de vrais réfugiés ? Les tensions morales dans la gesțion quotidienne de I'asile, in Didier Fassin et Jean-Sébastien Eideliman Éds., Économies morales contemporaines, Paris, La Découverte, pp. 155-173.

Mazzocchetti Jacinthe (2017) Mises en scène, souffrances et quêtes de dignité. Quelle humanité dans les parcours d'asile ?, in Andrea Ceriana Mayneri Dir., Entre errances et silences. Ethnographier des souffrances et des violences ordinaires, Louvain-la-Neuve, Academia, pp. 101-136.

Mazzocchetti Jacinthe (2014) Le corps comme permis de circuler. Du corpshéros au corps-souffrant dans les trajectoires migratoires et les possibilités de régularisation, Parcours anthropologique, 9, pp. 133-154.

Mazzocchetti Jacinthe (2008) Les arts de la parole comme appui à l'apprentissage du français et vecteurs de mieux-être pour le public demandeur d'asile, in Alphabétisation et français langue étrangère : modélisation d'un dispositif pluriel de formation auprès du public demandeur d'asile, Namur, Lire et Écrire Namur asbl, pp. 45-74.

Mbembe Achille (2016) Politiques de l'inimitié, Paris, La Découverte, 181 p.

Mekdjian Sarah (2016) Les récits migratoires sont-ils encore possibles dans le domaine des Refugee Studies ? Analyse critique et expérimentation de cartographies créatives, ACME: An International E-Journal for Critical Geographies, 15 (1), pp. 150-186.

Métraux Jean-Claude (2007) La connaissance, arrière-petite-fille de la reconnaissance mutuelle, Politiques sociales, 3 et 4 , pp. 60-78.

Monsutti Alessandro (2009) Itinérances transnationales : un éclairage sur les réseaux migratoires afghans, Critique internationale, 3 (44), pp. 83-104.

Ollivro Jean (2009) " La mondialité ", La distance, objet géographique, Atala, 12, pp. 187-213. 
Probst Johanna (2011) Entre faits et fiction : l'instruction de la demande d'asile en Allemagne et en France, Cultures \& Conflits, 84, [en ligne]. URL : http:// journals.openedition.org/conflits/18243

Ricœur Paul (2004) Parcours de la reconnaissance, Paris, Folio, 448 p.

Réseau Éducation sans Frontières (2015) DÉCLARÉ MAJEUR ! Les tests d'âge osseux, alibi " scientifique " de la chasse aux jeunes isolés étrangers, Paris, L’Harmattan, $70 \mathrm{p}$.

Vallet Cédric (2009) Test osseux, test douteux, Alter Échos, 285-286, pp. 32-33.

Valluy Jérôme (2006) Genèse du « faux-réfugié ", Plein droit, 2 (69), pp. 19-22.

Vanoeteren Alain et Gehrels Lys (2009) La prise en considération de la santé mentale dans la procédure d'asile, Revue du droit des étrangers, 155, pp. 492-543.

Vrancken Didier (2011) De la mise à l'épreuve des individus au gouvernement de soi, Mouvements, 65 , pp. 11-25.

Walia Harsha (2015) Démanteler les frontières. Contre l'impérialisme et le colonialisme, Québec, Lux Éditeur, 372 p.

Woodtli Patrick F. (2008) Entre ordre et chaos. Le corps biométrique, lieu du politique, Altérités, 5 (1), pp. 14-37. 


\section{Jacinthe Mazzocchetti}

\section{Dire la violence des frontières dans le rapport de force que constitue la procédure d'asile. Le cas d'Ali, de I'Afghanistan en Belgique}

Cet article a pour objet de décrire la violence des frontières, à la fois géopolitiques et symboliques, qui se donnent à voir au travers des procédures relatives aux demandes d'asile : demandes initiales, recours, refus, etc. À partir du récit de vie d'Ali Y., demandeur d'asile afghan (arrivé mineur en Belgique, mais non reconnu comme tel), ainsi que de l'ensemble des documents de son dossier (rapports d'auditions, recours, rapports d'experts, cartes géographiques officielles et mentales, etc.), l'article analyse l'ethnocentrisme des procédures d'asile au travers des questions que soulève l'usage du récit biographique dans I'examen des demandes d'asile (le fait de se raconter). II analyse également I'incidence sur l'issue de la procédure des représentations différenciées de l'espace, du temps et de la parenté du demandeur et de l'examinateur.

\section{Telling the Violence of Borders in the Power Relations that Constitutes the Asylum Process. The Case of Ali, from Afghanistan to Belgium}

The purpose of this paper is to describe the violence of the borders, both geopolitical and symbolic, which are seen through the procedures for asylum applications: initial applications, appeals, refusals, etc. From the narrative of life of Ali Y., an Afghan asylum seeker (who had arrived in Belgium but was not recognized as such), as well as all the documents in his file (reports of hearings, appeals, expert reports, geographical and mental maps, etc.), the article analyzes the ethnocentrism of asylum procedures through the questions raised by the use of the biographical narrative in the examination of asylum claims (telling oneself). It also analyses the impact on the outcome of the procedure of differentiated representations about space, time and kinship of the applicant and the examiner.

\section{Contar la violencia de las fronteras en el equilibrio de poder representado por el procedimiento de asilo. El caso de Ali, de Afganistán a Bélgica}

El propósito de este artículo es describir la violencia de las fronteras, tanto geopolíticas como simbólicas, que se ven a través de los trámites para las solicitudes de asilo: solicitudes iniciales, apelaciones, denegaciones, etc. De la narrativa de la vida de Ali Y., solicitante de asilo afgano (que había llegado a Bélgica pero no había sido reconocido como tal), así como todos los documentos de su expediente (informes de audiencias, apelaciones, informes de expertos, mapas oficial y mental, etc.), el artículo analiza el etnocentrismo de los procedimientos de asilo a través de las preguntas planteadas por el uso de la narrativa biográfica en el examen de las solicitudes de asilo. También analiza el impacto en el resultado de los procedimientos de las representaciones diferenciadas de espacio, tiempo y parentesco del solicitante y del examinador. 